\title{
Hybrid surgery for early gastric cancer
}

\author{
Osamu Goto ${ }^{1}$, Hiroya Takeuchi' ${ }^{2}$, Yuko Kitagawa $^{2}$, Naohisa Yahagi ${ }^{1}$ \\ ${ }^{1}$ Division of Research and Development for Minimally Invasive Treatment, Cancer Center, Keio University, School of Medicine, Tokyo, Japan; \\ ${ }^{2}$ Department of Surgery, Keio University, School of Medicine, Tokyo, Japan \\ Contributions: (I) Conception and design: O Goto; (II) Administrative support: N Yahagi; (III) Provision of study materials or patients: Y Kitagawa, \\ N Yahagi; (IV) Collection and assembly of data: O Goto, H Takeuchi; (V) Data analysis and interpretation: O Goto, H Takeuchi; (VI) Manuscript \\ writing: All authors; (VII) Final approval of manuscript: All authors. \\ Correspondence to: Osamu Goto, M.D., Ph.D. Division of Research and Development for Minimally Invasive Treatment, Cancer Center, Keio \\ University, School of Medicine, 35 Shinanomachi, Shinjuku-ku, Tokyo 160-8582, Japan. Email: ogotou-gi@keio.jp.
}

\begin{abstract}
Endoscopic submucosal dissection (ESD) is the most suitable treatment option in terms of minimally invasive treatment for potential node-negative early gastric cancers (EGCs). Furthermore, making the resection area of the primary lesion as small as possible is ideal for the patient's quality of life, even for potential node-positive EGC. An endoluminal approach is a reasonable option with which to minimize stomach resection area, because this procedure can be accurately demarcated from the inside. From this point of view, endoscopic full-thickness resection (EFTR) may be optimal, while laparoscopic assistance would be more desirable to create a more secure procedure. However, hybrid EFTR for EGCs has two limitations, which must be solved. First, concerns regarding iatrogenic tumor seeding via transluminal communication between the inside and outside of the tract exist. The second limitation relates to the determination of lymphadenectomy. Conventional lymphadenectomy, which involves the removal of the majority of feeding arteries, can lead to necrosis of the remaining gastric wall. Therefore, the resection area of lymphadenectomy should also be carefully determined. To address these two problems, a non-exposed hybrid EFTR combined with sentinel node navigation surgery (SNNS) would be the most ideal method of minimally invasive surgery for EGCs.
\end{abstract}

Keywords: Minimally invasive gastrectomy; endoscopic full-thickness resection (EFTR); non-exposure method; hybrid surgery; sentinel node navigation surgery (SNNS)

Received: 26 January 2016; Accepted: 04 March 2016; Published: 06 April 2016.

doi: $10.21037 / \operatorname{tgh} .2016 .03 .23$

View this article at: http://dx.doi.org/10.21037/tgh.2016.03.23

\section{Introduction}

Surgical resection is the first choice of treatment for gastric cancer with a high likelihood of a successful cure. Local resection is an accepted method for potential nodenegative resectable gastric cancers. Potential node-negative gastric cancers are early gastric cancers (EGCs), which have invaded the submucosal layers, and in such cases, an endoluminal approach can be applied. Histologically, intramucosal intestinal-type cancers irrespective of size; intramucosal intestinal-type cancers with ulcerative findings, which are $\leq 3 \mathrm{~cm}$ in size; submucosal intestinaltype cancers invading up to $500 \mu \mathrm{m}$ below the muscularis mucosae; and intramucosal diffuse-type cancers, which are $\leq 2 \mathrm{~cm}$ in size, are considered as node-negative cancers $(1,2)$ as long as the lesion can be resected in an en bloc fashion and has no angiolymphatic infiltration.

Endoscopic submucosal dissection (ESD) is an established endoscopic resection technique for EGCs (3-5), and many studies have demonstrated favorable and comparable outcomes for ESD $(6,7)$. While a randomized controlled trial is yet to be published, which directly compares ESD and surgery; it is clearly evident that ESD, which does not leave scars and can preserve the entire stomach, is likely to be far more superior to surgery in terms of the patient's quality of life. 
In contrast, potential node-positive EGCs include cancers that extensively invade the submucosal layer and must be resected in a full-thickness fashion to achieve secure en bloc resection. Furthermore, the treatment of potential node-positive EGCs should involve prophylactic dissection of lymph nodes where cancer cells might have metastasized. In this situation, laparoscopic assistance is required to perform a safe and secure resection. Here, we review of the current use of hybrid gastrectomy for EGCs and local lymphadenectomy and discuss its future perspectives.

\section{Endoscopic full-thickness resection (EFTR) with laparoscopic assistance}

With the emergence of natural orifice transluminal surgery (NOTES), laparoscopy-assisted EFTR became known as hybrid NOTES to differentiate this procedure from pure NOTES, which simply refers to endoscopic surgery without laparoscopic involvement (8-12). As pure NOTES is recognized as an advanced and challenging technique, particularly with regard to a transgastric approach that lacks the accessibility and reliability of a endoscopic closure method, the term hybrid NOTES is also uncommonly used. Nowadays, collaborative surgery, using both flexible endoscopy and rigid laparoscopy, is utilized in laparoscopic and endoscopic cooperative surgery (13) or in combined endoscopic and laparoscopic surgery (14).

EFTR with laparoscopic assistance for EGCs has been applied since the late 2000s. Abe et al. (15) reported the use of this procedure for an undifferentiated-type intramucosal cancer using the term laparoscopy-assisted EFTR. During this procedure, demarcation of the lesion and mucosal markings is performed by endoscopic observation, followed by endoscopic circumferential submucosal injection and ultimately, circumferential endoscopic mucosal incision. Subsequently, intentional perforation is executed on the exposed muscular layer and full-thickness resection is endoscopically performed under laparoscopic countertraction. After perorally or percutaneously retrieving the tumor, the full-thickness defect is then laparoscopically sutured. Park et al. (16) also demonstrated the feasibility of hybrid NOTES for EGC, which was simultaneously performed with laparoscopic colectomy. Cho et al. (17) further reported a case series of hybrid NOTES in which the surrounding lymph nodes were dissected.

Although EFTR with laparoscopic assistance is attractive with respect to organ preservation, avoidance of postoperative complications, and maintenance of the patient's quality of life, there remains an inevitable concern with regard to iatrogenic tumor seeding. By opening the gastric lumen during endoscopic intentional perforation and successive full-thickness resection, tumor cells floating in the gastric juice might be spread via spillage of the stomach contents or via transportation from the exposed surface of the primary lesion to the peritoneum by contact with laparoscopic instruments. Han et al. (18) observed tumor cells floating in approximately $15 \%$ of stomachs with EGCs. Hence, EFTR, which requires opening of the stomach, should not be deployed during the resection of epithelial neoplasms, which are exposed on the mucosal surface, or when patients possess subepithelial tumors (SETs) with ulcerative findings. In practice, the indication for EFTR with laparoscopic assistance is limited to SETs without ulceration.

\section{Non-exposure techniques for full-thickness resection}

To expand the indication of EFTR methods for cancers without the concern of iatrogenic dissemination by intentional perforation during the procedure, physicians have explored the use of non-exposure techniques for fullthickness resection. Laparoscopic wedge resection is a simple and reliable method. However, an unexpectedly large area has to be resected to achieve a secure R0 resection because demarcation of the lesion cannot be visualized from the outside of the lumen. Moreover, wedge resection using laparoscopic linear staplers leads to severe deformity of the remaining stomach, which might reduce the patient's quality of life in terms of food intake.

To minimize resection area during laparoscopic wedge resection, Inoue et al. (19) proposed the use of non-exposed full-thickness resection after seromuscular incision and referred to this as the combination of laparoscopic and endoscopic approaches to neoplasia with non-exposure technique (CLEAN-NET). In this technique, the full layers, including the lesion, are pulled to the peritoneal side after circumferential seromuscular incision and are resected using a linear stapler in the stretched mucosal or full layers. Prior to seromuscular incision, transluminal markings, which can be visible from the outside, are endoscopically made with the aid of a needle knife. Several transluminal sutures are then placed around the lesion to avoid dissociation of the layers. CLEAN-NET is technically accessible, but might become difficult depending on the location of the target lesion, e.g., the posterior wall of the upper third of the 
gastric body or fornix.

An ideal specimen that is resected by full-thickness resection would be an optimally demarcated full-layered resection without dissociation between the mucosal and serosal layers. To develop the non-exposure EFTR method, Goto et al. (20-22) proposed the use of nonexposed endoscopic wall-inversion surgery (NEWS). In this procedure, first, the resection area is first endoscopically demarcated with mucosal markings, followed by serosal markings under endoscopic navigation. Second, a circumferential seromuscular incision is laparoscopically performed, followed by endoscopic submucosal injection. Third, seromuscular layers are linearly sutured, with the lesion inverted into the inside. Finally, a muco-submucosal incision is endoscopically made, and the resected lesion is transorally retrieved. Using this technique, both mucosal and serosal planes can be optimally resected under direct visualization by endoscopy or laparoscopy. Although several issues need to be addressed, e.g., the technique requires skillful endoscopists and laparoscopists and is time consuming, this methodology is considered to be promising and is expected to develop as an ideal minimally invasive surgical procedure for EGCs in combination with sentinel node navigation surgery (SNNS) as mentioned later $(23,24)$.

Furthermore, other non-exposure methods have been introduced in animal models. For example, Kim et al. (25) demonstrated feasibility of the NEWS technique without seromuscular incision to omit a technically challenging phase in laparoscopy. This method may be simpler compared with NEWS, although the serosal area cannot be optimally demarcated and it is difficult to identify a suitable line to be cut at the phase of endoscopic resection for inverted lesion. It is clear that each procedure has its own pros and cons, and thus, further investigations are required to establish the method that is more accessible and clinically oriented.

Another advantage of the non-exposure method is that direct thermal damage of the serosal plane can be avoided. In pure EFTR, intentional perforation followed by seromuscular incision requires thermal effects, which can lead to unexpected damage of the organs outside of the stomach. In contrast to non-exposed EFTR, endoscopic intervention by electrocautery devices does not affect the extra-luminal space. From this point of view, non-exposed EFTR without laparoscopic assistance has also been developed. Schmidt et al. (26) introduced EFTR after lesion inversion by endoscopic suturing using a specially designed endoscopic suturing device. These authors successfully demonstrated the feasibility of this method for SETs in a small cases series. Takizawa et al. (27) further proposed the use of non-exposed EFTR using a sole flexible endoscope along with some commercially available devices by creating a circler mucosal tunnel around the lesion. Although these procedures are still unestablished, they may still become a promising, safe, and less-invasive method for endoluminal surgery.

\section{Local lymphadenectomy}

For treating cancers, non-exposed EFTR techniques would be appropriate for primary lesions to prevent possible tumor seeding. However, if these techniques are applied to patients, the indication for use is limited to only potential node-negative EGCs because no lymphadenectomy is considered. Because potential node-negative EGCs can be applied to ESD, the indication of EFTR is more restricted to potential node-negative EGCs that are technically difficult to resect by ESD, e.g., EGCs located at the gastric fundus or on the greater curvature at the upper third of the gastric body, or EGCs with severe scarring. To expand the indications for non-exposed EFTR to potential nodepositive EGCs, lymphadenectomy should be involved. However, standard lymphadenectomy might cause ischemia and necrosis of the remaining stomach because many lymph nodes lie alongside the major feeder arteries toward the stomach, and standard lymphadenectomy invariably involves the dissection of almost all feeders. Therefore, to perform local resection for potential node-positive cancers, the area of lymphadenectomy should also be localized and as many vessels as possible should be saved.

Regional lymphadenectomy can be an option to minimize the area of lymph node dissection. Seto et al. (28) proposed laparoscopic local resection combined with regional lymphadenectomy as a curative treatment option in a previous pilot study. Abe et al. (29) further introduced ESD followed by regional lymphadenectomy for EGC in which histological assessment revealed unexpectedly large undifferentiated cancer following ESD. In 2008, these authors proposed the use of hybrid EFTR followed by regional lymphadenectomy for potential node-positive EGCs (15). Cho et al. (17) further demonstrated the feasibility of the same method in 2011. Because there were no recurrences in these earlier reports, it appears that regional lymphadenectomy combined with local resection for a primary tumor appears to be acceptable, although large-scale assessments and long-term assessments are 


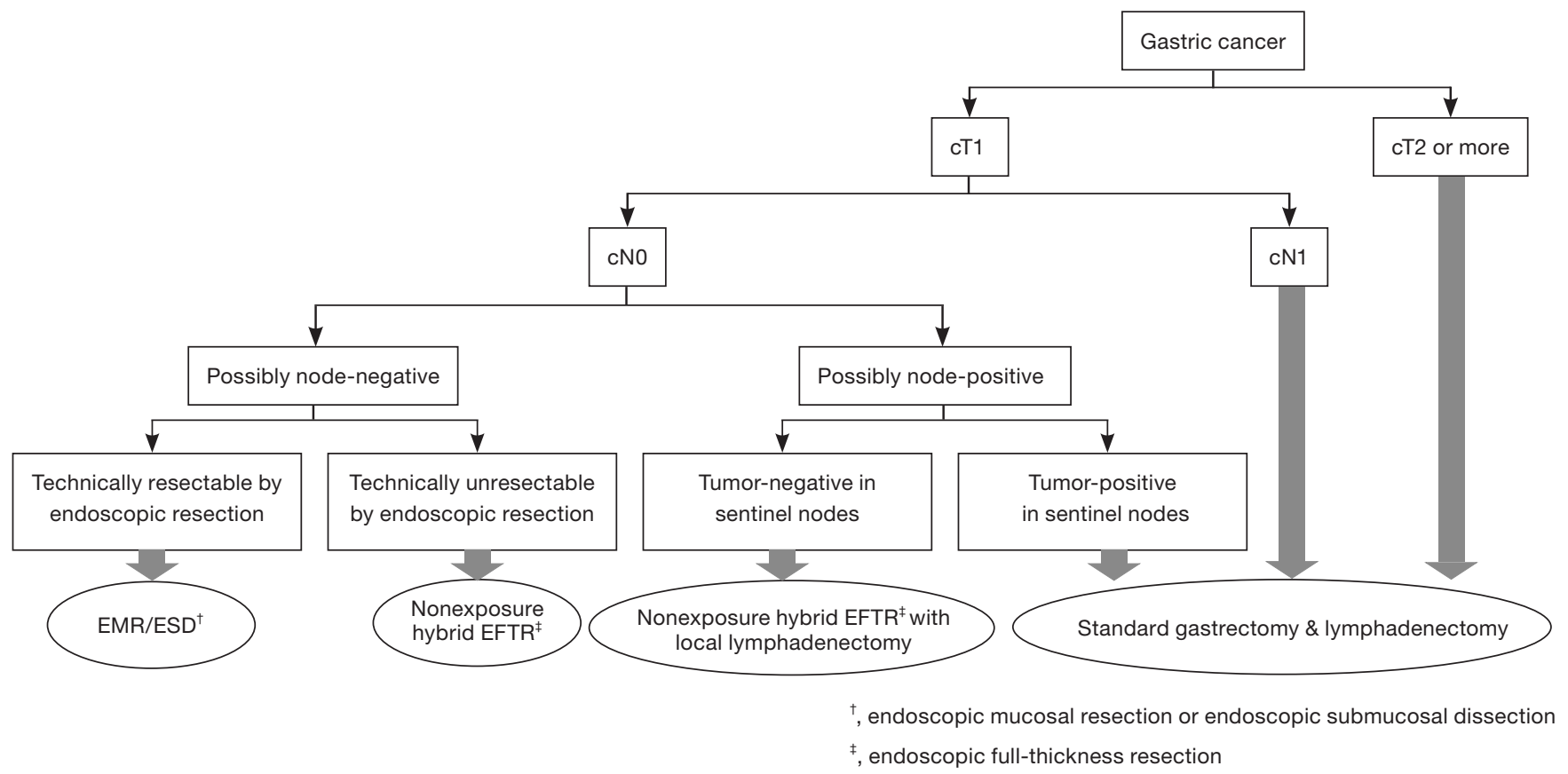

Figure 1 Advanced surgical approaches for gastric cancers.

urgently required.

However, there is no theoretical or statistical verification for the concept of regional lymphadenectomy. The area of regional lymph nodes for each type of cancer is empirically determined as the neighboring lymph node basin of the primary lesion. However, the precise location of the regional lymph basin receiving lymphatic flow from a tumor is difficult to accurately determine because the lymphatic network surrounding the stomach is complicated. In this regard, the sentinel node (SN) concept is likely to be more useful (30). If $\mathrm{SN}$, which represents the first drainage lymph node from the primary tumor, is found to be negative for cancerous cells, then it can be safely considered that no further metastases exist within the other lymph nodes. Lymphatic flow can be visualized by injecting a stained solution containing indocyanine green into the submucosal layer surrounding the lesion, and subsequently $\mathrm{SNs}$ are identified as lymph nodes that have been stained green or have shown radioactive accumulation by preoperatively injecting radioactive materials into the submucosal layer.

By proving that $\mathrm{SNs}$ are cancer free, the area of prophylactic lymphadenectomy can be readily minimized. Kitagawa et al. (31) aimed to validate the SN concept by investigating the distribution of SNs and tumor-positive lymph nodes in patients undergoing standard gastrectomy and conventional lymphadenectomy after intraoperatively investigating SNs and demonstrated the favorable diagnostic accuracy of lymph node metastasis using $\mathrm{SN}$ investigation.

After this study, we have started SNNS for EGCs, which are cT1N0M0, $\leq 4 \mathrm{~cm}$ in size, single lesion, and have not received any prior treatment. This technique is now offered by the Japanese government as a highly advanced medical treatment. Future studies will report regarding the clinical outcomes of this procedure.

\section{Future perspectives}

A flowchart depicting the advanced surgical approaches for gastric cancer is shown in Figure 1. Hybridization of endoscopy and laparoscopy has become established as less-invasive local resection for primary tumors. Furthermore, the combination of hybrid EFTR and local lymphadenectomy represents a useful curative treatment option for potential node-positive EGCs. In the current situation, the hybridization of non-exposed EFTR, e.g., NEWS and SNNS, would represent an ideal minimally invasive form of gastrectomy that can preserve function (24). However, there is still room for the development of both hybrid EFTR techniques and restricted lymphadenectomy concepts. The confirmation of favorable long-term survival is still required for the use of EFTR for primary lesion as along with the simplification and dissemination of these exciting 
procedures. Even in restricted lymphadenectomy navigated by the SN concept, the confirmation of long-term outcome in a large number of cases is still required to promote this new technique. Further investigations are vital if we are to establish the routine use of hybrid surgery for EGCs.

\section{Acknowledgements}

None.

\section{Footnote}

Conflicts of Interest: The authors have no conflicts of interest to declare.

\section{References}

1. Gotoda T, Yanagisawa A, Sasako M, et al. Incidence of lymph node metastasis from early gastric cancer: estimation with a large number of cases at two large centers. Gastric Cancer 2000;3:219-225.

2. Hirasawa T, Fujisaki J, Fukunaga T, et al. Lymph node metastasis from undifferentiated-type mucosal gastric cancer satisfying the expanded criteria for endoscopic resection based on routine histological examination. Gastric Cancer 2010;13:267-70.

3. Ono H, Kondo H, Gotoda T, et al. Endoscopic mucosal resection for treatment of early gastric cancer. Gut 2001;48:225-9.

4. Oyama T, Tomori A, Hotta K, et al. Endoscopic submucosal dissection of early esophageal cancer. Clin Gastroenterol Hepatol 2005;3:S67-70.

5. Yahagi N, Uraoka T, Ida Y, et al. Endoscopic submucosal dissection using the flex and the dual knives. Techn Gastrointest Endosc 2011;13:74-8.

6. Lian J, Chen S, Zhang Y, et al. A meta-analysis of endoscopic submucosal dissection and EMR for early gastric cancer. Gastrointest Endosc 2012;76:763-70.

7. Peng LJ, Tian SN, Lu L, et al. Outcome of endoscopic submucosal dissection for early gastric cancer of conventional and expanded indications: systematic review and meta-analysis. J Dig Dis 2015;16:67-74.

8. Nakajima K, Nishida T, Takahashi T, et al. Partial gastrectomy using natural orifice translumenal endoscopic surgery (NOTES) for gastric submucosal tumors: early experience in humans. Surg Endosc 2009;23:2650-5.

9. Rattner D, Kalloo A; ASGE/SAGES Working Group. ASGE/SAGES Working Group on Natural Orifice
Translumenal Endoscopic Surgery. October 2005. Surg Endosc 2006;20:329-33.

10. Kantsevoy SV. Endoscopic full-thickness resection: new minimally invasive therapeutic alternative for GI-tract lesions. Gastrointest Endosc 2006;64:90-1.

11. Shih SP, Kantsevoy SV, Kalloo AN, et al. Hybrid minimally invasive surgery--a bridge between laparoscopic and translumenal surgery. Surg Endosc 2007;21:1450-3.

12. Mohiuddin SS, Gonzalez JJ, Glass J, et al. Laparoscopicassisted endoluminal hybrid surgery: a stepping stone to NOTES. Surg Laparosc Endosc Percutan Tech 2009;19:474-8.

13. Hiki N, Yamamoto Y, Fukunaga T, et al. Laparoscopic and endoscopic cooperative surgery for gastrointestinal stromal tumor dissection. Surg Endosc 2008;22:1729-35.

14. Lee MK, Chen F, Esrailian E, et al. Combined endoscopic and laparoscopic surgery may be an alternative to bowel resection for the management of colon polyps not removable by standard colonoscopy. Surg Endosc 2013;27:2082-6.

15. Abe N, Mori T, Takeuchi H, et al. Successful treatment of early stage gastric cancer by laparoscopy-assisted endoscopic full-thickness resection with lymphadenectomy. Gastrointest Endosc 2008;68:1220-4.

16. Park S, Chun HJ, Keum B, et al. Successful hybrid NOTES resection of early gastric cancer in a patient with concomitant advanced colon cancer. Endoscopy 2010;42 Suppl 2:E1-2.

17. Cho WY, Kim YJ, Cho JY, et al. Hybrid natural orifice transluminal endoscopic surgery: endoscopic full-thickness resection of early gastric cancer and laparoscopic regional lymph node dissection--14 human cases. Endoscopy 2011;43:134-9.

18. Han TS, Kong SH, Lee HJ, et al. Dissemination of free cancer cells from the gastric lumen and from perigastric lymphovascular pedicles during radical gastric cancer surgery. Ann Surg Oncol 2011;18:2818-25.

19. Inoue H, Ikeda H, Hosoya T, et al. Endoscopic mucosal resection, endoscopic submucosal dissection, and beyond: full-layer resection for gastric cancer with nonexposure technique (CLEAN-NET). Surg Oncol Clin N Am 2012;21:129-40.

20. Goto O, Mitsui T, Fujishiro M, et al. New method of endoscopic full-thickness resection: a pilot study of nonexposed endoscopic wall-inversion surgery in an ex vivo porcine model. Gastric Cancer 2011;14:183-7.

21. Mitsui T, Goto O, Shimizu N, et al. Novel technique for full-thickness resection of gastric malignancy: feasibility 
of nonexposed endoscopic wall-inversion surgery (news) in porcine models. Surg Laparosc Endosc Percutan Tech 2013;23:e217-21.

22. Mitsui T, Niimi K, Yamashita H, et al. Non-exposed endoscopic wall-inversion surgery as a novel partial gastrectomy technique. Gastric Cancer 2014;17:594-9.

23. Goto O, Takeuchi H, Kawakubo H, et al. Feasibility of non-exposed endoscopic wall-inversion surgery with sentinel node basin dissection as a new surgical method for early gastric cancer: a porcine survival study. Gastric Cancer 2015;18:440-5.

24. Goto O, Takeuchi H, Kawakubo H, et al. First case of non-exposed endoscopic wall-inversion surgery with sentinel node basin dissection for early gastric cancer. Gastric Cancer 2015;18:434-9.

25. Kim CG, Yoon HM, Lee JY, et al. Nonexposure endolaparoscopic full-thickness resection with simple suturing technique. Endoscopy 2015;47:1171-4.

26. Schmidt A, Bauder M, Riecken B, et al. Endoscopic full-

doi: $10.21037 / \operatorname{tgh} .2016 .03 .23$

Cite this article as: Goto O, Takeuchi H, Kitagawa Y, Yahagi N. Hybrid surgery for early gastric cancer. Transl Gastroenterol Hepatol 2016;1:26. thickness resection of gastric subepithelial tumors: a single-center series. Endoscopy 2015;47:154-8.

27. Takizawa K, Knipschield MA, Gostout CJ. Submucosal endoscopy as an aid to full-thickness resection: pilot study in the porcine stomach. Gastrointest Endosc 2015;81:450-4.

28. Seto Y, Yamaguchi H, Shimoyama S, et al. Results of local resection with regional lymphadenectomy for early gastric cancer. Am J Surg 2001;182:498-501.

29. Abe N, Mori T, Izumisato Y, et al. Successful treatment of an undifferentiated early stage gastric cancer by combined en bloc EMR and laparoscopic regional lymphadenectomy. Gastrointest Endosc 2003;57:972-5.

30. Takeuchi H, Kitagawa Y. New sentinel node mapping technologies for early gastric cancer. Ann Surg Oncol 2013;20:522-32.

31. Kitagawa Y, Takeuchi H, Takagi Y, et al. Sentinel node mapping for gastric cancer: a prospective multicenter trial in Japan. J Clin Oncol 2013;31:3704-10. 